\title{
AVALIAÇÃO DA PREVALÊNCIA DE MASTITE SUBCLÍNICA EM OVELHAS
}

\section{Luan Sitó da Silva ${ }^{1}$, Karen Tiemi Akashi ${ }^{2}$, Stella Maris Teobaldo Tironi ${ }^{3}$, Jéssica} Priscila da Paz ${ }^{4}$, Antonio Campanha Martinez ${ }^{5}$

\author{
${ }^{1}$ Aluno do Programa de Mestrado em Produção Sustentável e Saúde Animal - UEM, \\ contato: luan-sito08@hotmail.com \\ ${ }^{2}$ Médica veterinária e bolsista do Projeto de Extensão/Fundação Araucária - UEM \\ ${ }^{3}$ Mestre em Produção Sustentável e Saúde Animal e bolsista do Projeto de \\ Extensão/Fundação Araucária - UEM \\ ${ }^{4}$ Aluna do curso de Medicina Veterinária - UEM \\ ${ }^{5}$ Professor do departamento de Medicina Veterinária - DMV/UEM
}

\section{Recebido em: 06/04/2019 - Aprovado em: 10/06/2019 - Publicado em: 30/06/2019} DOI: 10.18677/EnciBio_2019A23

\begin{abstract}
RESUMO
A ovinocultura encontra-se em constante crescimento mundialmente. Nessa atividade, a mastite é uma das enfermidades que acometem as ovelhas, gerando desmame de cordeiros leves, ou até mesmo a perda definitiva da função da glândula mamária. Sendo assim, seu diagnóstico deve ser precoce, evitando-se essas perdas na produção. Nesse estudo, foram avaliadas 413 ovelhas, destas 300 se apresentavam lactantes e foram inseridas nas avaliações. Para isso, foram coletados $2 \mathrm{~mL}$ de amostra de leite de cada glândula mamária e adicionados $2 \mathrm{~mL}$ de reagente do California Mastitis Test (CMT). Após homogeneização, a reação foi interpretada, considerando-se 5 graus de aglutinação: (-) sem alteração de consistência, (+) presença de gel ou formas espessas de lodo, mas sem mobilidade, $(++)$ forma espessa de gel grumoso, com movimentação em direção ao centro, (+++) formas de gel distintas com adesão à parte inferior da raquete para CMT e (MC: mastite clínica) consistência grumosa, mesmo sem adição do reagente. $O$ objetivo desse estudo foi realizar um levantamento da prevalência de mastite subclínica em ovelhas na região metropolitana de Umuarama-PR. Das propriedades avaliadas, $90 \%$ continha animais positivos. Dentre os animais avaliados, 55,6\% apresentaramse positivos no teste, com uma variação de 0 a $90,4 \%$ entre as propriedades. Quanto à mastite clínica, houve incidência de 2 a $34 \%$ de acometimento dos animais entre as propriedades. Sendo assim, conclui-se que a mastite clínica e subclínica são enfermidades de grande acometimento dos animais presentes nas propriedades de criação dessa espécie.
\end{abstract}

PALAVRAS-CHAVE: Enfermidade, glândula, leite, mamária, ovinos 


\title{
PREVALENCE OF SUBCLINICAL MASTITIS IN EWES
}

\begin{abstract}
Sheep farming is constantly growing worldwide. In this activity, mastitis is one of the diseases that affects the sheep, generating weaning of light lambs, or even the definitive loss of function of the mammary gland. Therefore, its diagnosis should be early, avoiding such losses in production. In this study, 413 sheep were evaluated, of which 300 were presented as infants and were inserted in the evaluations. For this, $2 \mathrm{~mL}$ of milk sample was collected from each mammary gland and $2 \mathrm{~mL}$ of California Mastitis Test (CMT) reagent was added. After homogenization, the reaction was interpreted, considering 5 degrees of agglutination: (-) without change of consistency, $(+)$ presence of gel or thick sludge forms, but without mobility, $(++)$ thick form of lump gel, with movement towards the center, $(+++)$ distinct gel forms with adhesion to the lower part of the CMT racket and (MC: clinical mastitis) lump consistency, even without addition of the reagent. The objective of this study was to conduct a survey of the prevalence of subclinical mastitis in sheep in the metropolitan region of Umuarama-PR. Of the evaluated properties, $90 \%$ contained positive animals. Among the animals evaluated, $55.6 \%$ were positive in the test, with a variation of 0 to $90.4 \%$ between the properties. As for clinical mastitis, there was a 2 to $34 \%$ incidence of involvement of the animals between the properties. Therefore, it is concluded that clinical and subclinical mastitis are diseases of great affection of the animals present in the breeding properties of this species.
\end{abstract}

KEYWORDS: Disease, gland, mammary, milk, sheep.

\section{INTRODUÇÃO}

A produção de ovinos está em significante desenvolvimento no Brasil, com destaque no Paraná (MEXIA et al., 2011). O rebanho brasileiro atingiu o valor de 17.976.367 ovinos no Brasil em 2017 (IBGE, 2017). O principal produto exportado pelo Brasil é o item lã suja, representando quase $57 \%$ da exportação total de produtos ovinos. O principal parceiro comercial em 2017 foi o Uruguai, com participação de 60,7\%, exclusivamente composta por lã. O segundo parceiro comercial foi a Itália, cujos principais produtos comercializados são pele ou couro (EMBRAPA, 2017).

Apesar da evolução mencionada, existem alguns fatores limitantes ao crescimento desta atividade, dentre eles, os fatores sanitários. Uma das enfermidades que mais se destaca é a mastite. Esta acomete ovelhas em todo o mundo, entretanto, os estudos nesse contexto são mais numerosos nos países onde estes animais são criados para a produção leiteira (BERGONIER et al., 2003; CONTRERAS et al., 2007).

Sabe-se que esse mal altera a capacidade funcional da glândula mamária, acometendo-a parcial ou totalmente, podendo até levar o animal à morte. Desta forma, a mastite influência direta e negativamente a produtividade do rebanho, acarretando perdas econômicas ao produtor (PEREIRA et al., 2014).

Além disso, a doença diminui a produção e a qualidade do leite, gerando cordeiros mais leves, de crescimento reduzido ou mortes por inanição. Por fim, a mastite clínica ou subclínica gera aumento de custos com cuidados veterinários e medicamentos, sendo a principal causa do descarte precoce de ovelhas, pela perda de função da mama acometida (JONES; WATKINS, 2000; MENZIES; RAMANOON, 2001). 
A mastite subclínica é uma forma da doença que está intimamente relacionada com a forma mais severa da doença, a mastite clínica. Isso ocorre pois, sabe-se que existe uma grande ocorrência de evolução da enfermidade (ZAFALON et al., 2018a). O objetivo desse estudo foi estabelecer a prevalência de mastite clínica e subclínica em ovelhas, na região metropolitana de Umuarama-PR.

\section{MATERIAL E MÉTODOS}

Os manejos empregados durante este trabalho foram realizados de acordo a atender aos princípios éticos e de bem-estar animal. Como parte integrante do projeto "Produção Sustentável e Estruturação da Cadeia de Ovinos de Corte", financiado pelo Programa Universidade Sem Fronteiras da Secretaria de Ciência e Tecnologia do Estado do Paraná, foram coletados dados de dez propriedades de ovinocultura na região metropolitana de Umuarama - PR, entre abril de 2017 a março de 2018.

Inicialmente, foi realizada a anamnese com o produtor, de modo a identificar animais que não estavam em lactação ou que estavam em estágio final de gestação. Feito isso, foi realizado o exame físico geral dos animais, através da inspeção e palpação da glândula mamária, onde detectava-se aumento de volume, assimetria das glândulas, presença de nódulos ou endurecimento da glândula, demonstrando perda da função da mesma. Foram avaliadas 413 ovelhas, destas 300 se apresentavam lactantes e foram inseridos nas avaliações.

Para o diagnóstico da mastite subclínica (MS) foi realizado o teste de california mastitis test (CMT), onde coletou-se $2 \mathrm{~mL}$ de leite de forma manual em placa de plástico com 4 poços e $2 \mathrm{~mL}$ do reagente comercial para CMT (CMT Solução, Tadabrás ${ }^{\circledR}$, Brasil) foi adicionado ao mesmo poço, e essas amostras eram homogeneizadas sempre nessa proporção, através de movimentos circulares da placa.

Para diagnóstico de mastite clínica, o leite era coletado da mesma maneira, porém detectava-se visualmente consistência grumosa. De acordo com a técnica de Barnum e Newbould (1961), o diagnóstico foi interpretado pelo aspecto visual da reação, em que foram considerados 5 graus de aglutinação, sendo eles: Negativo (): seria sem alteração de consistência; 1 cruz (+): gel ou formas espessas de lodo, mas quando a pá é girada a solução não se move para o centro; 2 cruzes $(++)$ : forma espessa de gel grumoso, que, quando girada, move-se rapidamente em direção ao centro; 3 cruzes (+++): formas de gel distintas que tendem a aderir a parte inferior da raquete de CMT, e durante o turbilhão um pico central distinto se forma; Mastite clínica (MC): consistência grumosa mesmo sem adição do reagente.

As interpretações foram realizadas sempre pelos mesmos avaliadores. $\mathrm{E}$ os animais que apresentaram mastite clínica não foram avaliados pelo método de reação com o CMT, já que o diagnóstico foi definitivo logo na retirada da amostra do leite.

\section{RESULTADOS E DISCUSSÃO}

Dentre as propriedades avaliadas, 9 das 10 propriedades (90\%) apresentaram animais que continham pelo menos um dos tipos de mastite, conforme apresentado na TABELA 1. 
TABELA 1. Relação de propriedades, animais avaliados e exames realizados e animais positivos para mastite subclínica (MS) por cidade.

\begin{tabular}{ccccc}
\hline CIDADE & PROPRIEDADES & ANIMAIS & $\begin{array}{c}\text { EXAMES } \\
\text { REALIZADOS }\end{array}$ & $\begin{array}{c}\text { POSITIVOS } \\
\text { (MS) }\end{array}$ \\
\hline A & 6 & 232 & 173 & 96 \\
B & 1 & 64 & 41 & 24 \\
C & 2 & 113 & 82 & 45 \\
D & 1 & 4 & 4 & 2 \\
\hline TOTAL & 10 & 413 & 300 & 167 \\
\hline
\end{tabular}

Dos 300 animais, $133(44,4 \%)$ não apresentaram nenhum tipo de mastite, considerando-se saudáveis. Nenhuma propriedade, realizava o exame periódico das mamas, em nenhum momento importante como, parição, secagem e antes da estação de monta. Pereira et al. (2014) observaram que em 59,2\% das propriedades não era realizado nenhum exame periódico das mamas nos momentos recomendados. Uma das medidas importantes para casos novos e crônicos, o que é essencial para eliminação das fontes de infecção e diminuir a transmissão da doença dentro do rebanho (CONTRERAS et al., 2007).

\section{MASTITE SUBCLÍNICA}

A mastite subclínica, tem uma prevalência média estimada nos rebanhos leiteiros europeus variando de 20 a 30\% (BERGONIER; BERTHELOT, 2003), o que se parece com as frequências observadas nas ovelhas de corte da raça Santa Inês criadas no Brasil (COUTINHO et al., 2006; DOMINGUES et al., 2006; GUARANÁ et al., 2011).

Dentre os exames realizados, onde se descontam animais com mastite clínica e animais secos, houve incidência de 0 a $90,4 \%$ de mastite subclínica entre as propriedades, com uma média de $55,6 \%$ entre elas. Os resultados diferem de Vasileiou et al. (2018) na Grécia e Santana et al. (2016), que avaliaram 2.220 e 307 ovelhas, onde apenas $26 \%$ e $29,6 \%$, respectivamente, estavam positivas para mastite subclínica, em pelo menos uma das metades das glândulas mamárias

Em outro estudo, Zafalon et al. (2017) observaram 65\% dos animais positivos para mastite subclínica. Estudos de Zafalon et al. (2018b) avaliaram 60 ovelhas Santa Inês e Morada Nova e encontraram 100\% dos animais contendo pelo menos um dos lados da glândula mamária das ovelhas afetado. Estudos realizados em Portugal em 2017, em 18 rebanhos, observou-se uma incidência de 32,2\% de mastite subclínica em ovelhas (QUEIROGA, 2017). Estudo recente, observou uma incidência de $22,6 \%$ de animais acometidos com mastite subclínica (KORDALIS et al., 2019). Esses resultados demonstram que a mastite subclínica está presente em quantidade significativa no rebanho ovino, assim como, os dados encontrados em nosso trabalho.

O animal acometido pela enfermidade diminui a produção de leite, e segundo Pereira et al. (2014), 92\% das propriedades acometidas havia uma redução do ganho de peso dos cordeiros lactentes, e em $70 \%$ observou-se uma diminuição e até mesmo ausência da produção de leite da matriz, levando a morte dos cordeiros neonatos, além disso, nesse estudo foi observada baixa escrituração zootécnica de dados relacionados à mortalidade de neonatos, o que dificulta a realização do controle produtivo do rebanho, já que a mastite pode ser uma delas. Dos animais 
avaliados pelo teste CMT, não houve diferença significativa com relação ao lado do teto mais acometido, como demonstrado na FIGURA 1.

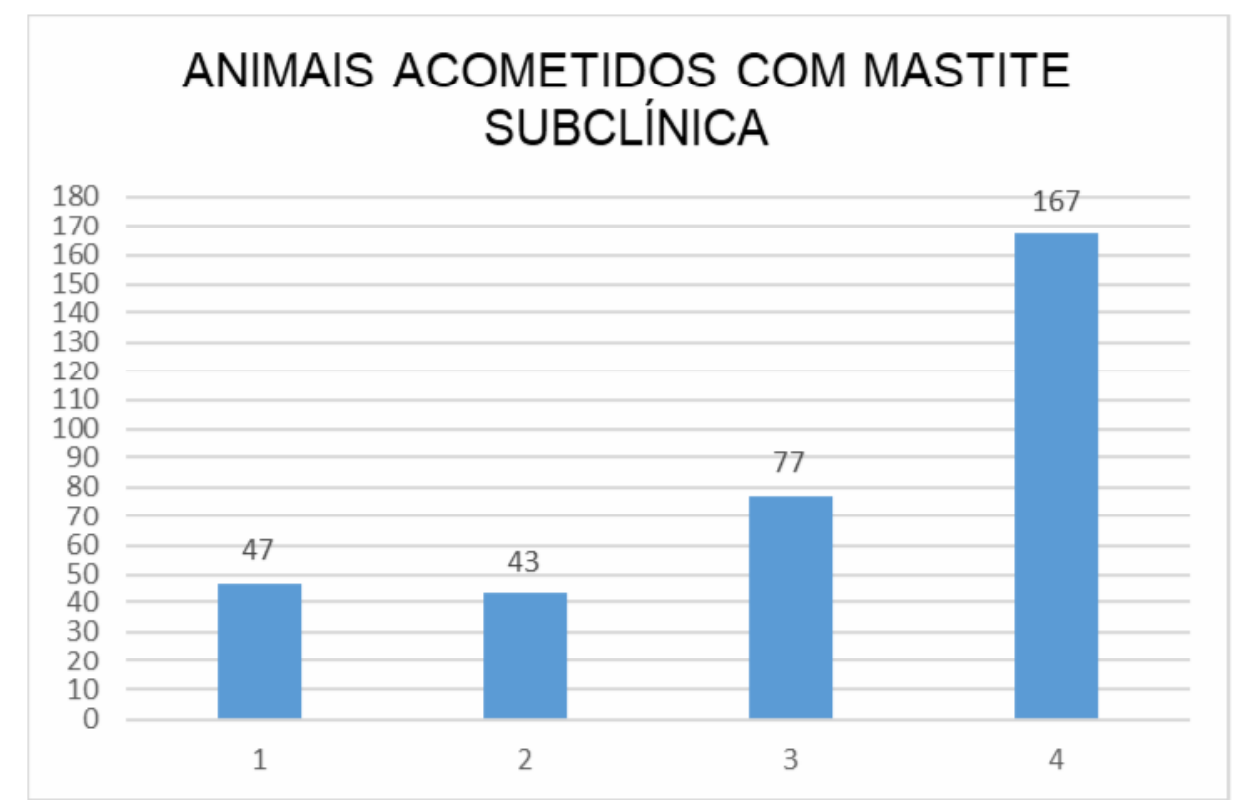

FIGURA 1. Número de animais cometidos com mastite subclínica. 1) Quantidade de animais com somente o teto esquerdo acometido; 2) quantidade de animais com somente o teto direito acometido; 3) quantidade de animais com os dois tetos acometidos; 4) quantidade total de animais com mastite subclínica.

Zafalon et al. (2015) utilizando 160 ovelhas, observou que 63 mamas estavam acometidas por mastite subclínica. Zafalon et al. (2016) utilizando 479 ovelhas Santa Inês e 342 Morada nova, totalizando 821 metades, observou que 55,8\% das partes apresentavam mastite subclínica.

Estudos recentes na Grécia, demonstraram que a ordenha manual foi um fator predisponente para a mastite subclínica em ovelhas (VASILEIOU et al., 2018) Outro estudo recente procurando a incidência de mastite positiva para Streptococcus sp. em ovinos e caprinos observaram uma incidência de $29,20 \%$ e $31,20 \%$ respectivamente (HAGGAG et al., 2019)

\section{MASTITE CLÍNICA}

Quanto à mastite clínica, 44 animais (12,8\%) apresentaram-se positivos (TABELA 2), havendo uma incidência de 2 a 34\% entre as propriedades.

TABELA 2. Quantidade de animais avaliados por região e positivos para mastite clínica (MC).

\begin{tabular}{ccccc}
\hline CIDADE & PROPRIEDADES & ANIMAIS & $\begin{array}{c}\text { EXAMES } \\
\text { REALIZADOS }\end{array}$ & $\begin{array}{c}\text { POSITIVOS } \\
\text { (MC) }\end{array}$ \\
\hline A & 6 & 232 & 174 & 18 \\
B & 1 & 64 & 41 & 1 \\
C & 2 & 113 & 82 & 25 \\
D & 1 & 4 & 4 & 0 \\
\hline TOTAL & 10 & 413 & 300 & 44 \\
\hline
\end{tabular}


Esses dados estão de acordo com Pereira et al. (2014) que, avaliando 54 rebanhos de ovelhas do norte do Paraná, observaram frequência de 2 a $30 \%$ de mastite clínica nos rebanhos. Os resultados são próximos de Queiroga (2017), em Portugal, onde houve uma incidência de 1,7\% de mastite clínica em 18 rebanhos estudados.

Entretanto, quanto ao lado mais acometido para mastite clínica, assim como na subclínica, também não houve diferença, como demonstrado na FIGURA 2, que representa a quantidade de animais acometidos por mastite clínica e qual teto foi mais acometido.

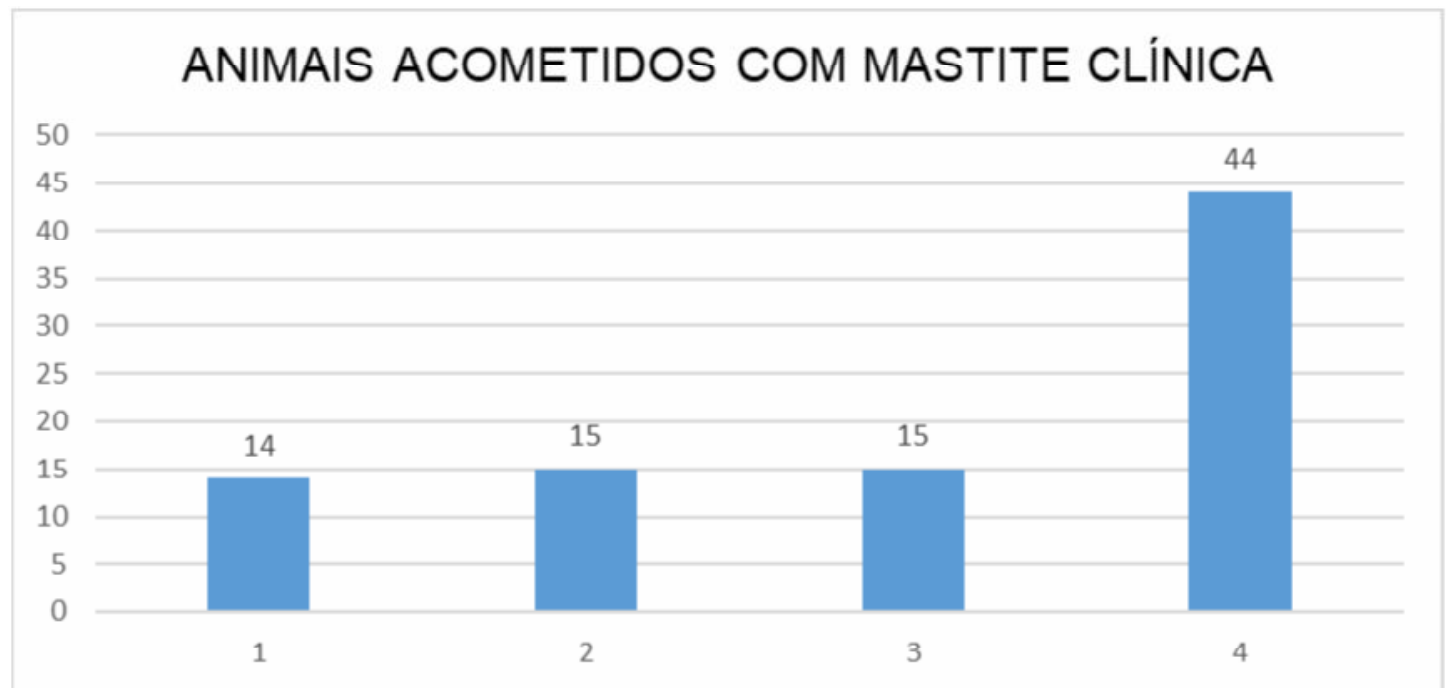

FIGURA 2. Número de animais acometidos com mastite clínica. 1) Quantidade de animais com somente o teto esquerdo acometido; 2) quantidade de animais com somente o teto direito acometido; 3) quantidade de animais com os dois tetos acometidos; 4) quantidade total de animais acometidos com mastite clínica.

\section{MASTITE CLÍNICA X MASTITE SUBCLÍNICA}

Houve diferença entre a relação de animais acometidos com mastite subclínica e mastite clínica, como demonstrado na FIGURA 3, estando a mastite clínica presente em menor quantidade, porém, de forma mais severa.

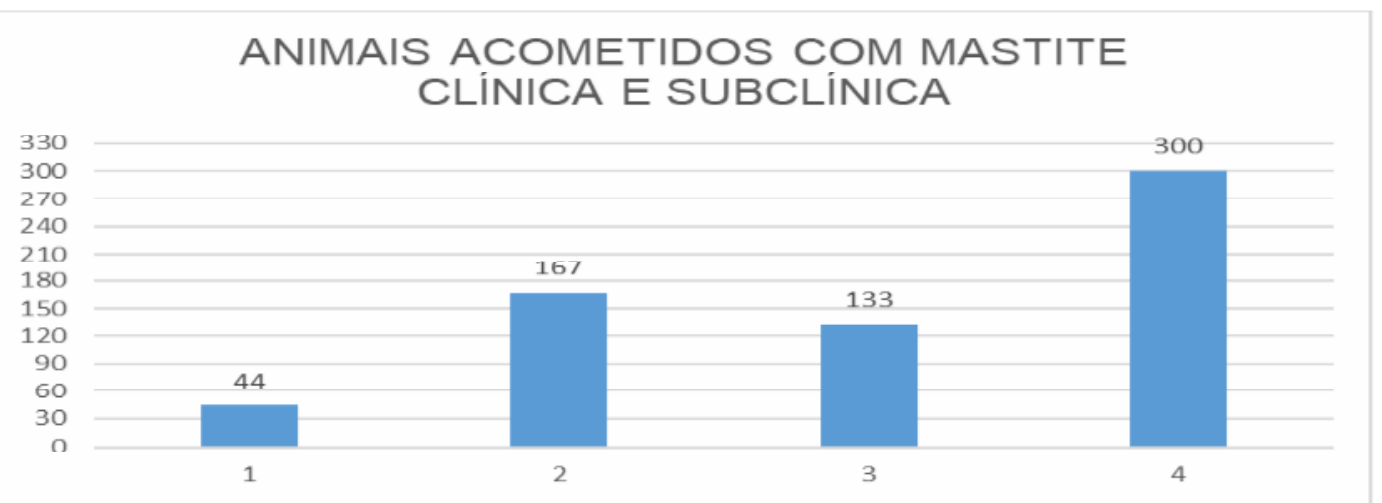

FIGURA 3. Número de animais acometidos com mastite clínica e subclínica nos rebanhos.

1) Quantidade de animais acometidos com mastite clínica; 2) quantidade de animais com subclínica;

3) quantidade de animais saudáveis; 4) total de animais submetidos ao CMT. 
Os produtores atendidos pelo experimento desconheciam a presença da enfermidade em seu rebanho, portanto, não foi instituído nenhum tipo de tratamento prévio às análises. No estudo realizado por Pereira et al. (2014) das propriedades acometidas pela enfermidade, $56,4 \%$ dos proprietários instituíram algum tipo de tratamento, sendo que $13,7 \%$ das propriedades só utilizavam antimicrobianos intramamários, $27,3 \%$ somente antimicrobianos parenterais e 59\% utilizavam associações de parenterais e intramamários.

Acredita-se que a baixa taxa de sucesso no tratamento pode ser atribuída ao caráter irreversível das lesões do parênquima mamário, na maioria dos casos, ou ao início tardio do tratamento, e ainda, a utilização dos antimicrobianos era inadequada, na maioria das vezes, em doses erradas, com tempo de administração curto, e com troca frequente de princípios ativos. Aos animais com grau 3 de mastite subclínica e mastite clínica foi sugerido aos produtores realizar o tratamento.

\section{CONCLUSÃO}

Conclui-se que a mastite subclínica afeta uma quantidade significante de ovelhas, podendo ambos os tetos serem afetados. Já com relação à mastite clínica, verificou-se menor incidência dessa enfermidade nos animais quando comparada à forma subclínica.

\section{REFERÊNCIAS}

BARNUM, D. A.; NEWBOULDT, F. H. S. The use of the california mastitis test for the detection of bovine mastitis. The Canadian Veterinary Journal, v.2, n. 3, p. 83-90, 1961. Disponível em:< https://www.ncbi.nlm.nih.gov/pmc/articles/PMC1585631/>. Acesso em: 28/03/2019.

BERGONIER, D.; BERTHELOT, X. New advances in epizootology and control of ewe mastitis. Livestock Production Science, v. 79, n. 1, p. 1-16, 2003. Disponível em: $\quad<$ https://doi.org/10.1016/S0301-6226(02)00145-8>. DOI: 10.1016/S03016226(02)00145-8. Acesso em: 28/03/2019.

BERGONIER, D.; CRÉMOUX, R.; RUPP, R.; LAGRIFFOUL, G.; BERTHELOT, X. Mastitis of dairy small ruminants. Veterinary Research, v. 34, n. 5, p.689-716, 2003. Disponível em: <https://www.ncbi.nlm.nih.gov/pubmed/14556701>. DOI: 10.1051/vetres:2003030. Acesso em: 28/03/2019.

CONTRERAS, A.; SIERRA, D.; SÁNCHEZ, A.; CORRALES, J.C.; MARCO, J.C., et al. Mastitis in small ruminant. Small Ruminant. Research, v.68, n.2, p.145-153, 2007. Disponível em: :10.1016/j.smallrumres.2006.09.011. Acesso em: 28/03/2019.

COUTINHO, D.A.; COSTA, J.N.; RIBEIRO, M.G.; TORRES, J.A. Etiologia e sensibilidade antimicrobiana in vitro de bactérias isoladas de ovelhas da raça Santa Inês com mastite subclínica. Revista Brasileira de Saúde e Produção Animal, v. 7, n. 2, p. 139-151, 2006. Disponível em: $<$ https://repositorio.ufba.br/ri/bitstream/ri/1908/1/712-2767-2-PB.pdf>. Acesso em: 28/03/2019. 
DOMINGUES P.F., LUCHEIS S.B., SERRÃO L.S., FERNANDES S., CONTENTE A.P.A., MARTINS E.C.V. \& LANGONI H. Etiologia e sensibilidade bacteriana da mastite subclínica em ovelhas da raça Santa Inês. Ars Veterinaria, v. 22, n. 2, p.146- 152, $2006 . \quad$ Disponível em: <http://arsveterinaria.org.br/index.php/ars/article/view/94>.DOI: http://dx.doi.org/10.1 5361/2175-0106.2006v22n2p146-152. Acesso em: 15/03/2019.

EMBRAPA. Ovinocultura e Caprinocultura Conjuntura econômica, aspectos produtivos de 2017 e perspectivas para 2018. Boletim do Centro de Inteligência e Mercado de Caprinos e Ovinos, n. 2, p. 1-17, 2017. Disponível em: < https://www.infoteca.cnptia.embrapa.br/infoteca/bitstream/doc/1097193/1/CNPC2017 Ovinoculturaecaprinocultura.pdf>. Acesso em: 28/03/2019.

GUARANÁ E.L.S., SANTOS R.A., CAMPOS A.G.S.S., SILVA N.S., AFONSO J.A.B., et al. Dinâmica celular e microbiológica do leite de ovelhas Santa Inês acompanhadas durante a lactação. Pesquisa Veterinária Brasileira, v.31, n.10, p.851-858, 2011. Disponível em: <http://www.scielo.br/scielo.php?pid=S0100736X2011001000004\&script=sci_abstract\&tlng=pt>. DOI: $\quad 10.1590 /$ S0100736X2011001000004. Acesso em: 28/03/2019.

HAGGAG, Y.; NOSSAIR, M.; NAGGAR, A.; ABDALLAH, M.; HEBA, H., et al. Microbiological Studies on Streptococcus Species Associated with Environmental Mastitis in Sheep and Goats Farms. Alexandria Journal of Veterinary Sciences, v.60, n.1, p.155-160, 2019. $\quad$ Disponível $\quad$ em: https://www.ejmanager.com/mnstemps/31/31-1539167653.pdf?t=1558189008 DOI: 10.5455/ajvs.12105. Acesso em: 28/03/2019.

Instituto Brasileiro de Geografia e Estatística - IBGE. Pesquisa de Pecuária Municipal. 2017. Disponível em: <https://www.ibge.gov.br/estatisticasnovoportal/economicas/agricultura-e-pecuaria/9107-producao-da-pecuariamunicipal.html?=\&t=resultados $>$. Acesso em: 08 Nov. 2018.

JONES, J.E.T. \& WATKINS, G.H. Mastitis and contagious agalactia. In: Martin W.B. \& Aitken I.D. (Eds), Diseases of Sheep. 3rd ed. Blackwell Science, UK, p.75- 80, 2000. Disponível em: https://s3.amazonaws.com/academia.edu.documents/40702055/Disease_of_Sheep 4th_Edition_Edited_By_I.pdf?AWSAccessKeyld=AKIAIWOWYYGZ2Y53UL 3 A\&Expir es $=1558192847 \&$ Signature $=$ TEO1s\%2BB2wGp\%2FIShfmJYehnv4JOM\%3D\&respo nse-content-

disposition=inline\%3B\%20filename\%3Ddiseaes_of_sheep.pdf\#page=115. Acesso em: 28/03/2019.

KORDALIS, N.G.; ARSENOPOULOS, K.; VASILEIOU, N.G.C.; MAVROGIANNI, V.S.; LIANOU, D.T., et at. Field evidence for association between increased gastrointestinal nematode burden and subclinical mastitis in dairy sheep. Veterinary Parasitology, v. 265, p. 56-62, 1 jan. 2019. Disponível em: <https://www.sciencedirect.com/science/article/pii/S0304401718303820 >. DOI: 10.1016/j.vetpar.2018.11.010. Acesso em: 27 mar. 2019. 
MENZIES, P.I. \& RAMANOON, S. Mastitis of sheep and goats. Veterinary Clinics of North America: Food Animal Practice, v. 17, n. 2, p.333-358, 2001. Disponível em: < https://www.sciencedirect.com/science/article/pii/S0749072015300323>. DOI: 10.1016/S0749-0720(15)30032-3. Acesso em: 02/03/2019.

MEXIA, A. A.; DE MACEDO, F. A. F.; DE OLIVEIRA, C. A. L.; ZUNDT, M.; YAMAMOTO, S. M., et al. Susceptibilidade a nematóides em ovelhas Santa Inês, Bergamácia e Texel no Noroeste do Paraná. Semina: Ciências Agrárias, Londrina, v. $\quad 32, \quad$ supl. 1, p. 1921-1928, 2011. Disponível em:< http://www.uel.br/revistas//uel/index.php/semagrarias/article/view/5723>. DOI: 10.5433/1679-0359.2011v32n4Sup1p1921. Acesso em: 28/03/2019.

PEREIRA, P. F. V.; STOTZER, E. S.; PRETTOGIORDANO, L. G.; MÜLLER, E. E.; LISBÔA, J. A. N. Fatores de risco, etiologia e aspectos clínicos da mastite em ovelhas de corte no Paraná. Pesquisa Veterinária Brasileira, Rio de Janeiro, v. 34, n. 1, p. 1-10, 2014. Disponível em:< http://www.scielo.br/scielo.php?pid=S0100736X2014000100001\&script=sci_abstract\&tIng=es >.DOI:10.1590/S0100736X20140 00100001 . Acesso em: 28/03/2019.

QUEIROGA, M.C. Prevalence and aetiology of sheep mastitis in Alentejo region of Portugal. Small Ruminant Research, v. 153, p. 123-130, 1 ago. 2017. Disponível em: < https://www.sciencedirect.com/science/article/pii/S0921448817301621 >.DOI: 10.1016/j.smallrumres.2017.06.003 Acesso em: 26 mar. 2019.

SANTANA, R. C. M.; ZAFALON, L. F.; BRANDÃO, H. M.; JUNIOR, G.A.F.; PILON, L. E., et al. Uso de antimicrobiano nanoparticulado para o tratamento da mastite subclínica de ovelhas de corte no período seco. Pesquisa Veterinária Brasileira, v.36, n.9, p.826-830, 2016. Disponível em: < http://www.scielo.br/pdf/pvb/v36n9/1678-5150-pvb-36-09-00826.pdf>. $\quad$ DOI: 10.1590/S0100-736X2016000900006. Acesso em: 28/03/2019.

VASILEIOU, N. G. C.; CRIPPS, P. J.; IOANNIDI, K. S.; CHATZOPOULOS, D. C.; GOUGOULIS, D. A., et al.Extensive countrywide field investigation of subclinical mastitis in sheep in Greece. Journal Dairy Science, v.101, p.7297-7310, 2018. Disponível em: <https://www.ncbi.nlm.nih.gov/pubmed/29859691>. DOI: 10.3168/jds.2017-14075 Acesso em: 28/03/2019.

VASILEIOU, N. G. C.; CHATZOPOULOSA, D.C.; GOUGOULISA, D.A.; SARROUB, S.; KATSAFADOUA, A.I., et al. Slime-producing staphylococci as causal agents of subclinical mastitis in sheep. Veterinary Microbiology, v. 224, p. 93-99, 1 out. 2018. em:

https://www.sciencedirect.com/science/article/pii/S0378113518308952>.

DOI:10.1016/j.vetmic.2018.08.022. Acesso em: 28/03/2019.

ZAFALON, L. F.; CUNHA, M. L. R. S.; RIBOLI, D. F. M.; PILON, L.E. Persistência de staphylococcus coagulase-negativos em glândulas mamárias de ovelhas com mastite subclínica após o tratamento antimicrobiano à secagem. Ciência Animal Brasileira, Goiânia, v.18, p.1-11, 2017a. Disponível em: < http://www.scielo.br/scielo.php?pid=S1809- 
68912017000100321\&script=sci_abstract\&tlng=pt>. DOI: $10.1590 / 1089-6891 \mathrm{v} 18 \mathrm{e}-$ 44328. Acesso em: 28/03/2019.

ZAFALON, L. F.; CUNHA, M.L.R.S.; BRANDÃO, H. M.; MOSQUEIRA, V.C.F.; SANTANA, R.C.M., et al. Relationship between virulence factor genes in coagulasenegative Staphylococcus spp. and failure of antimicrobial treatment of subclinical mastitis in sheep. Pesquisa Veterinária Brasileira, v.38, n.4, p.579-585, 2018a. Disponível em: <http://www.scielo.br/scielo.php?script=sci_arttext\&pid=S0100736X2018000400579\&lng=en\&tlng=en >.DOl:10.1590/1678-5150-pvb-4984>. Acesso em: 28/03/2019.

ZAFALON, L. F.; FIM JÚNIOR, G. A.; VASOI, C. O.; LOPES, N. S. S.; VESCHI, J. L. A., et al. Influence of lactation stages and rain periods on subclinical mastitis in meat producing ewes. Ciência Rural, v.46, n.10, p.1797-1803, 2016. Disponível em: $<$ http://www.scielo.br/scielo.php?script=sci_arttext\&pid=S0103-

84782016001001797>. DOI: 10.1590/0103-8478cr20151068. Acesso em: 28/03/2019.

ZAFALON, L. F.; VESCHI, J. L. A.; MARTINS, K. B.; SANTANA, R. C. M. Ocorrência de mastite subclínica ovina durante duas lactações consecutivas em rebanho da raça santa inês. Ciência Animal Brasileira, v.16, n.1, p. 116-124, 2015. Disponível em: $<$ http://www.scielo.br/scielo.php?pid=S1809 68912015000100116\&script=sci_abstract\&tlng=pt>. DOI: $\quad$ 10.1590/108968916i120779. Acesso em: 28/03/2019.

ZAFALON, L.F.; SANTANA, R.C.M.; ESTEVES, S.N.; FIM JÚNIOR, G.A. Contagem de células somáticas para o diagnóstico da mastite subclínica ovina em diferentes raças. Semina: Ciências Agrárias, Londrina, v. 39, n. 4, p. 1555-1564, $2018 \mathrm{~b}$. Disponível em: <http://www.uel.br/revistas/uel/index.php/semagrarias/article/view/27028> DOI: 10.5433/1679-0359.2018v39n4p1555. Acesso em: 28/03/2019. 